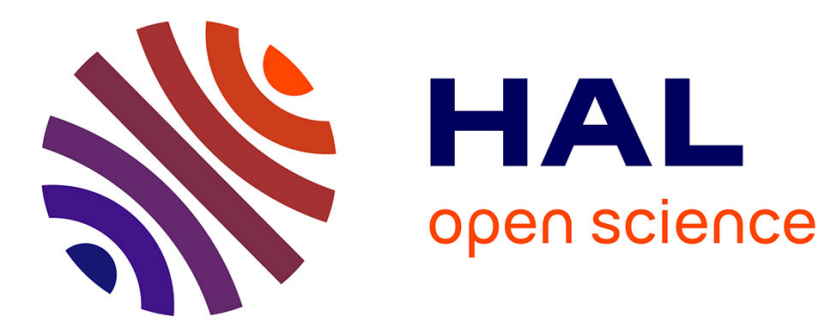

\title{
On-Wafer Measurement Errors Due to Unwanted Radiations on High-Q Inductors
}

Olga Bushueva, Christophe Viallon, Ayad Ghannam, Thierry Parra

\section{To cite this version:}

Olga Bushueva, Christophe Viallon, Ayad Ghannam, Thierry Parra. On-Wafer Measurement Errors Due to Unwanted Radiations on High-Q Inductors. IEEE Transactions on Microwave Theory and Techniques, 2016, 64 (9), pp.2905-2911. 10.1109/TMTT.2016.2588486 . hal-01370500

\section{HAL Id: hal-01370500 https://hal.science/hal-01370500}

Submitted on 22 Sep 2016

HAL is a multi-disciplinary open access archive for the deposit and dissemination of scientific research documents, whether they are published or not. The documents may come from teaching and research institutions in France or abroad, or from public or private research centers.
L'archive ouverte pluridisciplinaire HAL, est destinée au dépôt et à la diffusion de documents scientifiques de niveau recherche, publiés ou non, émanant des établissements d'enseignement et de recherche français ou étrangers, des laboratoires publics ou privés. 


\title{
On-Wafer Measurement Errors due to Unwanted Radiations on High-Q Inductors
}

\author{
Olga Bushueva, Christophe Viallon, Member, IEEE, Ayad Ghannam, and Thierry Parra
}

\begin{abstract}
This paper investigates the disagreements that may occur between on-wafer measurements and electromagnetic (EM) simulations of high- $Q$ inductive devices. Such disagreements are highlighted on a planar spiral inductor and a 3-D solenoid which exhibit measured maximum Q-factors of 27 and 35 respectively, while 42 and 45 were expected from EM simulations. Both devices are fabricated on high-resistivity substrates. A radiative interaction is identified between $R F$ probe and inductive device under test. By using EM simulations, extra-losses associated with this parasitic effect are fully modeled through the calculation of radiation and dissipation related Q-factors. Adjustments of onwafer probe setup are proposed to reduce this parasitic effect. Finally, the 3-D solenoid inductor is characterized using a new experimental fixture, and the maximum $Q$ of 45 predicted by EM simulation is retrieved.
\end{abstract}

Index Terms-On-wafer measurements, quality factor, high-Q inductor, high-resistivity substrate, $\mathrm{RF}$ probe, radiation.

\section{INTRODUCTION}

$\mathbf{T}$ HE increasing demand for miniaturized, efficient and low-cost microwave integrated systems requires the development of new technologies offering high-Q interconnections and inductive passive devices. The performance evaluation of such device is usually performed on-wafer by using a vector network analyzer (VNA) along with coaxial cables and RF probes. A high level of accuracy is reached by first removing the contribution of the measurement test-bench up to the end of the RF probe tips [1]. This calibration process involves well-proven methods such as Short-Open-LoadThru (SOLT). As a second step, specific on-wafer standards can be also measured to deembed the influence of pads and interconnections surrounding the device-under-test (DUT). Measurement reference planes are then shifted toward DUT terminals.

However, even if the previous calibration process is performed carefully, some errors may remain when characterizing high-Q RF inductors. Mismatches between measurements and simulations may be observed which are usually resulting from the probing setup surrounding the inductor. These disagreements depend on the nature of the problem and they are noticed on the shape of the extracted Q-factor versus frequency, with unexpected ripples and/or resonances. Several parasitic phenomena leading to characterization errors have

This work is supported by Région Midi-Pyrénées (Project Millitechno3D, grant CLE-2012 no. 12050932) and by LAAS-CNRS micro and nano technolgies platform member of the French RENATECH network.

C. Viallon, T. Parra and O. Bushueva are with LAAS-CNRS and University of Toulouse, UPS, LAAS, 7 avenue du Colonel Roche, F-31031 Toulouse, France, e-mail: (cviallon@laas.fr; parra@laas.fr).

A. Ghannam is with 3DiS Technologies, 478 rue de la Découverte, CS 67624, 31676 Labège, France, e-mail: (ayad.ghannam@3dis-tech.com). been already identified. In [2], a return current path difference to the nearby ground through a high-resistive substrate is observed. RF probes can also stimulate a second propagating mode along with the chuck that couples to the DUT at some specific situations [3], [4]. At millimeter-wave frequencies, the RF probe can couple with the DUT and adjacent test structures enabling new conduction paths [5], [6].

In this paper, a parasitic radiative interaction between the RF probe and the on-wafer measured structure is investigated and fully analyzed. As a parasitic dipole antenna structure is set up, Q-factor values from experiment are well below values extracted from EM simulations. This issue has been observed above few $\mathrm{GHz}$, when the Q-factor of inductors exceeds the range 25 to 35 . It is demonstrated in Section II for both inductors, 3-D solenoid and planar spiral integrated on glass or high-resistivity silicon (HR-Si). But it has been noticed also for LTCC inductors [1].

Some EM analyses including the RF probe are carried out on the same devices, as detailed in Section III. The implementation of the RF probe significantly improves the agreement between simulations and measurements. Plotted radiation patterns suggest that measured Q-factors are underestimated because of EM radiations caused by this interaction between RF probe and DUT.

Section IV explains how to quantitatively determine the weight of all parasitic phenomena from the EM field calculated within the simulated volume. Dissipation and radiation contributions are then separated into the overall Q-factor.

In the last section, several approaches are explored to almost remove the radiation. They are based on a better confinement of the field by properly setting the DUT surrounding metal ring.

\section{Illustration OF Dis AGREEMENTS BetWEen SIMULATION AND MEASUREMENT}

Inductors used for the demonstration of this issue are described below. The first one is a 4-turn $3 \mathrm{nH} 3-\mathrm{D}$ solenoid (Fig. 1). It has been fabricated either on $\mathrm{HR}-\mathrm{Si}(4.5 \mathrm{k} \Omega \mathrm{cm})$ or on glass substrate using a low-cost single-step 3-D copper electroplating process [7] marketed by 3DiS Technologies company. Metal conductor is $13 \mu \mathrm{m}$-thick and $30 \mu \mathrm{m}$-wide. Turn spacing is $120 \mu \mathrm{m}$, and the upper part is placed on top of $100 \mu \mathrm{m}$-thick, $60 \mu \mathrm{m}$-wide and $360 \mu \mathrm{m}$-long SU8 pillars. Further results are related to the HR-Si version. The second device is a 3-turn $7.1 \mathrm{nH}$ planar spiral inductor fabricated on HR-Si substrate using a standard planar process (Fig. 1). Spiral dimensions and process cross-section are given in Fig. 2. 

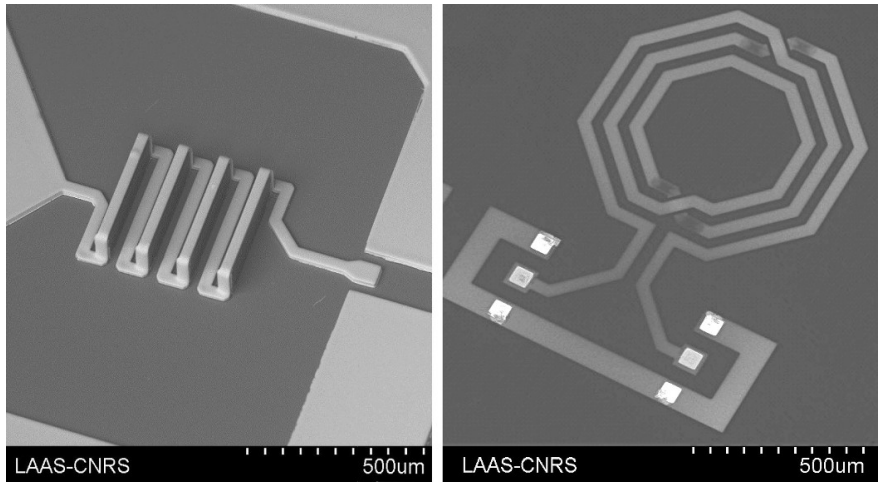

Fig. 1. Microphotographs of the $3 \mathrm{nH}$ 3-D solenoid (left) and the $7.1 \mathrm{nH}$ planar spiral inductor (right).

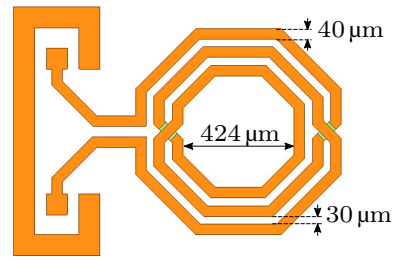

(a)

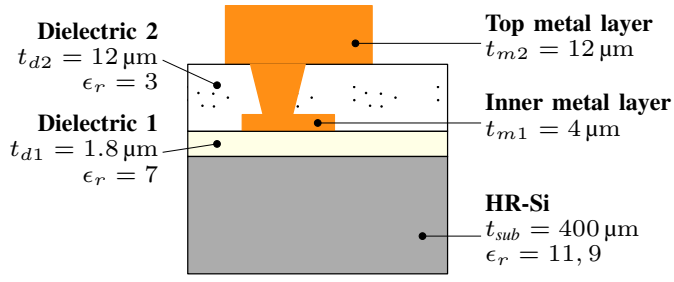

(b)

Fig. 2. (a) Top view and (b) schematic cross-section of the $7.1 \mathrm{nH}$ planar spiral inductor.

$S$-parameters measurements are carried out using an Anritsu vector network analyser (VNA) MS4647A and a Cascade Microtech Infinity Ground-Signal-Ground (GSG) RF probe with a pitch of $150 \mu \mathrm{m}$ (Fig. 3). A SOLT calibration is performed prior to measurements in order to set the reference plane at the end of probe tips. The accuracy of the test bench has been carefully checked by repeating several calibrations and measurements on the same DUT, using other VNA, cables, probes and calibration standards. A worst-case error magnitude of 0.002 was observed on the reflection coefficient of the 3-D solenoid all over the measured frequency range from $400 \mathrm{MHz}$ to $20 \mathrm{GHz}$. At $5 \mathrm{GHz}$, this value translates into an error of about $6 \%$ on a Q-factor of 30 [1], which is quite acceptable. RF pads have not been deembedded to avoid any additional measurement error. EM simulations are performed using the 3-D EM simulation software HFSS from ANSYS. Internal excitation ports are drawn between signal pad and ground to keep the same location as for measurements.

Measured and simulated $S$-parameters of inductors are converted to $Y$-parameters. The quality factor $Q$ and inductance value $L$ are then extracted from the admittance $Y_{11}$ using (1) and (2). For a two-port inductor, this formulation considers that port 2 is shorted.

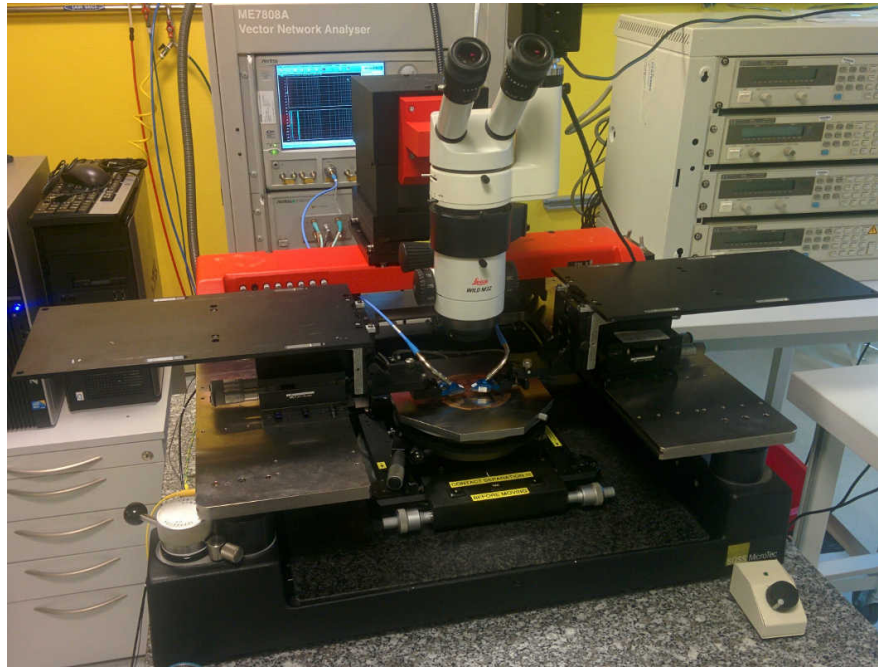

Fig. 3. Photograph of the measurement set-up.

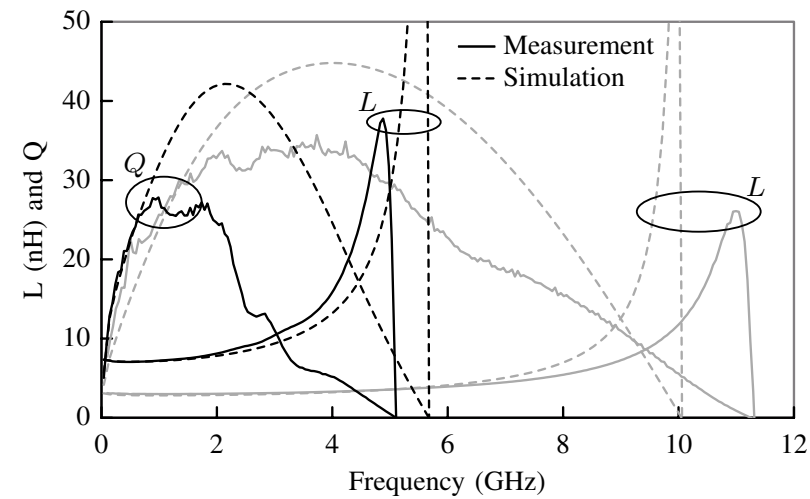

Fig. 4. Measured and simulated Q-factor and inductance value of both 3-D solenoid (grey curves) and planar spiral inductor (black curves).

$$
\begin{gathered}
Q=-\frac{\operatorname{Im}\left(Y_{11}\right)}{\operatorname{Re}\left(Y_{11}\right)} \\
L=\frac{\operatorname{Im}\left(Y_{11}^{-1}\right)}{\omega}
\end{gathered}
$$

Q-factor and inductor values from simulations and measurements are displayed in Fig. 4 for both inductors. Good agreement between inductance and Q-factor values can be noticed as long as losses are mainly governed by skin and proximity effects, and located into conductors (below $1 \mathrm{GHz}$ for the planar spiral and $2 \mathrm{GHz}$ for the solenoid). However, a large mismatch is observed on Q-factors when frequency is increasing and on self-resonant frequencies (SRF). Simulated Q-factor values (42 and 45) are respectively $29 \%$ and $56 \%$ highers than measured ones (27 and 35). Such differences cannot be explained by the $6 \%$ measurement uncertainty.

The solenoid presented in Fig. 1 has been fabricated on a glass wafer. It is characterized using the previous test bench and, this time, two other $150 \mu \mathrm{m}$ GSG RF probes are also used, i.e., a Cascade Z-probe Z67 and a MPI T40. Extracted Q-factors (Fig. 5) show that previously observed mismatches are probe-dependant and are still much larger than stated 


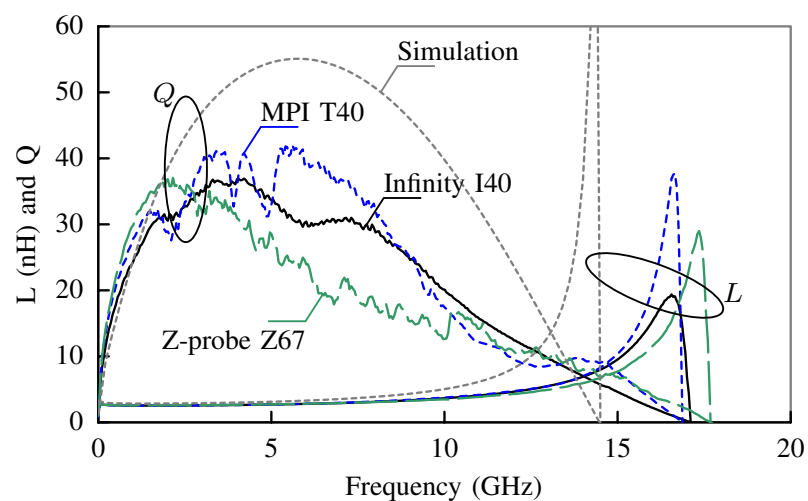

Fig. 5. Influence of RF probes on the extracted Q-factor and inductance values of the $3 \mathrm{nH} 3-\mathrm{D}$ solenoid inductor fabricated on a glass substrate.

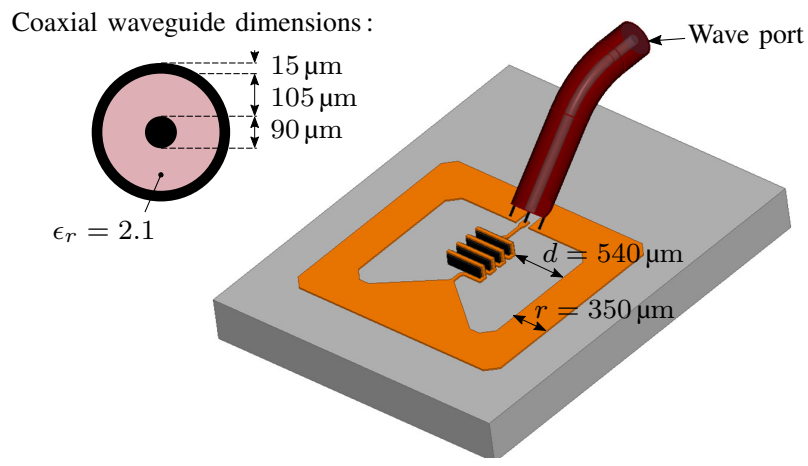

Fig. 6. 3-D modeling of the solenoid inductor including RF probe.

measurement uncertainty. Main differences between all those RF probes are the final part of the feeding waveguide and its elevation angle. The Z67 probe is based on a tapered coplanar waveguide sloped by $30^{\circ}$ while $45^{\circ}$ coaxial waveguides are used on T40 and Infinity RF probes. This result point out an interaction between RF probes and the DUT. In following sections only the Infinity RF probe is considered but observations and conclusions have been verified to be the same for the other probes.

\section{EM SimULATIONS INCLUdING RF PROBE}

A model of the Cascade Infinity RF probe is added into the EM modeling to improve the agreement of this modeling with the measurement environment, and to investigate interactions that occur between the RF probe and DUT. Only the final part of the feeding coaxial waveguide beyond the absorber shielding is added into the simulation box (Fig. 6). All dimensions are given in Fig. 6. They have been extrapolated from the approximate measure of the external diameter of the coaxial waveguide to fit with a $50 \Omega$ characteristic impedance (manufacturer does not provide such data). Probe tips are simplified, as well, since it can be considered that their length $(100 \mu \mathrm{m})$ is small enough when compared with wavelength values under $15 \mathrm{GHz}$. The elevation angle of the actual probe from the horizontal plane is respected. The bend of the coaxial waveguide noticeable in Fig. 6 is added only to reach the lateral face of the simulation box where a wave-port is drawn.

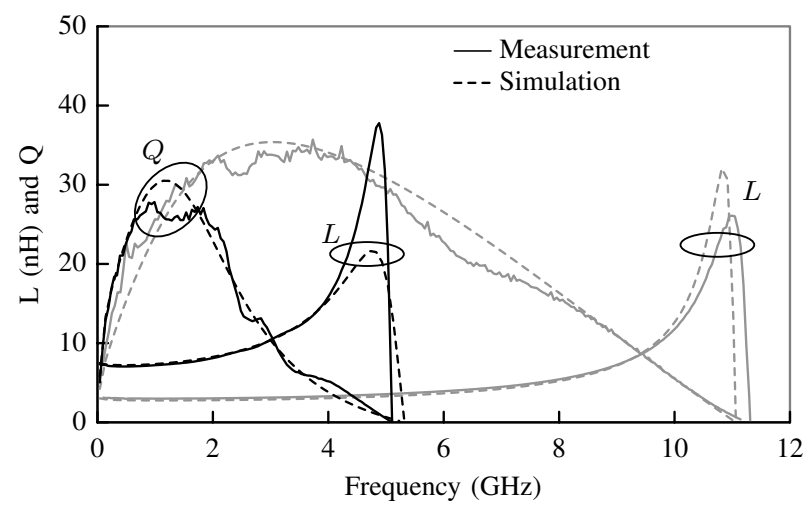

Fig. 7. Measured Q-factor and inductance value of both 3-D solenoid (grey curves) and planar spiral inductor (black curves) compared with simulation results carried out when an RF probe model is used.

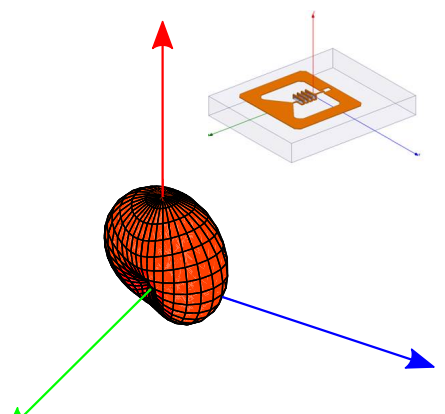

(a) Internal port

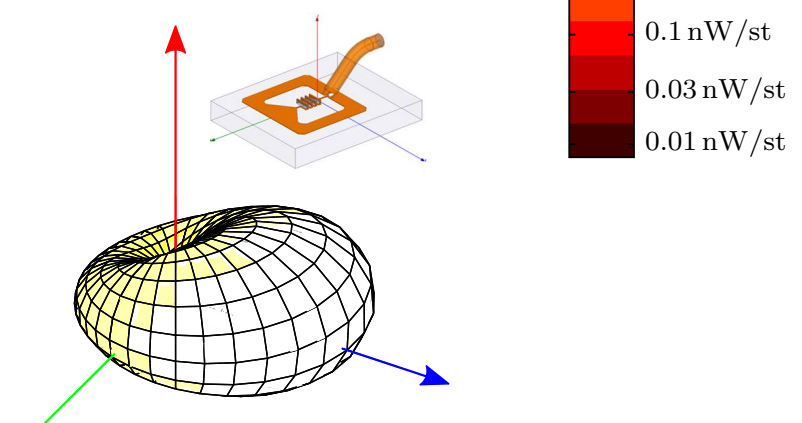

(b) RF probe

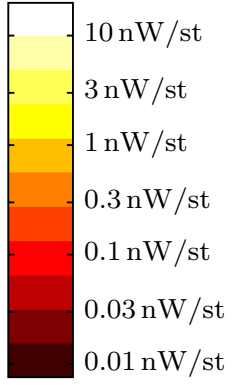

Fig. 8. Radiation intensity at $5 \mathrm{GHz}$ of the solenoid inductor expressed in nWatt/steradian. The excitation port of the DUT is successively (a) internal and (b) external through an RF probe.

The coaxial waveguide and probe tips are deembedded from simulations as a comparison with measurements is performed.

Measured and newly simulated $L$ and $Q$ values are compared in Fig. 7 for both solenoid and planar inductors. As it can be noticed, agreements are now quite good for both devices. Then, this new EM environment can be used to investigate the interaction between the DUT and the RF probe.

3-D radiation intensity patterns are calculated at $5 \mathrm{GHz}$ for the solenoid inductor, when the device is excited either with an internal port [Fig. 8(a)], and also with an RF probe [Fig. 8(b)]. It can be noticed that the radiated power intensity is more than one order of magnitude higher in all directions when the RF probe is included within simulation. 
As a conclusion, it can be stated that the degradation observed on the experimental Q-factor results from excessive radiations occurring in the $\mathrm{GHz}$ range. The 3-D radiation intensity from [Fig. 8(b)] is quite similar to what can be observed for a dipole antenna placed above a reflector plane. The two branches of the dipole would be the DUT structure including the surrounding metal ring and the external conductor of the feeding coaxial waveguide beyond the absorber shielding, whereas the chuck forms the reflector.

All on-wafer characterizations we have done on several planar and 3-D inductors ranging from 1 to $10 \mathrm{nH}$ show that this effect is noticeable when Q-factor values exceed the range 25 to 35 and when frequencies are above 1 or $2 \mathrm{GHz}$. It is masked when characterizing high-turn inductors because of limited SRF values and/or proximity effects which lower Qfactors. For smaller inductors, this radiation effect should be still present above $20 \mathrm{GHz}$, but it may be hidden by increasing skin effect losses. The radiated power depends on the inductor topology, as well, as on dimensions and technology of RF probes. The geometry of the inductor's surrounding metal ring also affects radiation losses as we will see in Section V.

\section{Quantitative Evaluation of The Losses of AN INDUCTOR}

\section{A. Extraction of the Powers from Simulated $E$ and $H$ Fields}

Radiated and accepted input powers, $P_{\text {rad }}$ and $P_{a c c}$ respectively, can be computed from EM simulation [8]. Thanks to the energy conservation law, the power dissipated by the inductor $\left(P_{\text {diss }}\right)$ into the volume is deduced from $P_{\text {rad }}$ and $P_{a c c}$ using the following relationship

$$
P_{a c c}=P_{\text {diss }}+P_{\text {rad }}
$$

For the 3-D solenoid, values of these powers are reported in Table I at a frequency of $5 \mathrm{GHz}$ and for both internal and RF probing EM excitations. It can be noticed that $P_{\text {diss }}$ remains unchanged in both cases whereas radiated power $P_{\text {rad }}$ is over 20 times higher when the RF probe is included into simulation. $P_{a c c}$ increases only by the excess amount of $P_{\text {rad }}$. Data of Table I confirm that excess losses observed at $5 \mathrm{GHz}$ when the RF probe is present are mainly resulting from radiations.

This analysis demonstrates that when the power dissipated into the inductor is low, as it is for high-Q inductor, the radiated power can be no longer negligible. In this case, it can become quite difficult to deduce the actual performance of the inductor from on-wafer measurements. As a consequence, special care must be brought in designing the characterization fixture. This design requires an accurate modeling of the whole structure formed when the inductor is under test. This modeling is developed in the following paragraphs.

\section{B. Extraction of the Stored Energy from $E$ and $H$ Fields}

Stored electric and magnetic energies, $W_{e}$ and $W_{m}$, can also be computed from vectors of electric field $\boldsymbol{E}$ and magnetic field $\boldsymbol{H}$, simulated within each volume defined within the simulation box [8]. The stored energy $W_{\text {sto }}$ of an inductor is defined as follows [9]

$$
W_{s t o}=W_{m}-W_{e}
$$

TABLE I

ACCEPTED, RAdiATED AND Dissipated POWERS OF THE 3-D SOLENOID AT $5 \mathrm{GHz}$

\begin{tabular}{llll}
\hline \hline Inductor & $\begin{array}{l}P_{\text {acc }} \\
(\mu \mathrm{W})\end{array}$ & $\begin{array}{l}P_{\text {rad }} \\
(\mu \mathrm{W})\end{array}$ & $\begin{array}{l}P_{\text {diss }} \\
(\mu \mathrm{W})\end{array}$ \\
\hline Simulation with internal port & 340 & 8 & 332 \\
Simulation with RF probe & 512 & 171 & 341 \\
\hline \hline
\end{tabular}

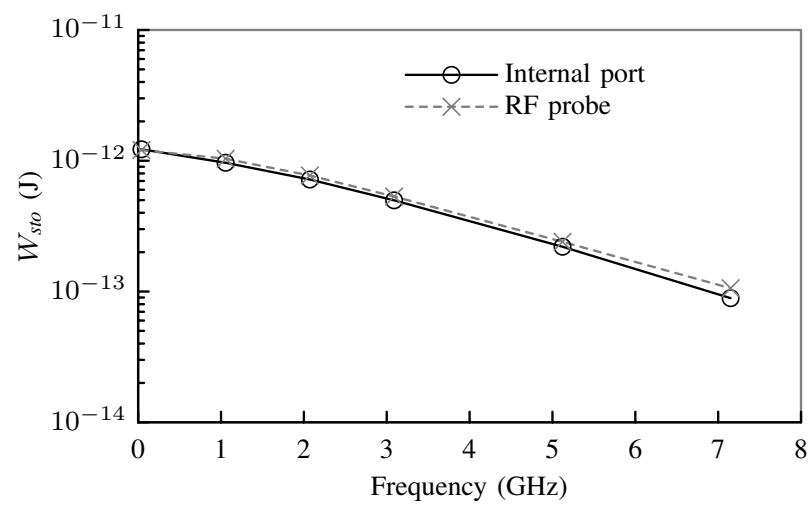

Fig. 9. Stored energy in the $3 \mathrm{nH}$ Solenoid calculated from simulations carried out using an internal port and then an RF probe.

Extracted $W_{\text {sto }}$ energy versus frequency is shown in Fig. 9, for both previous excitation ways (i.e., with an internal port or with an RF probe). Both simulations lead to the same $W_{\text {sto }}$ values which confirm that the same inductor is simulated in both cases. This last result means that both simulations could be compared from powers $P_{a c c}, P_{\text {rad }}$ and $P_{\text {diss }}$. However, Qfactors are introduced for next developments as they can apply for components with different values.

\section{Modeling with $Q$-factors}

The Q-factor of a reactive device gives energy losses relatively to the stored energy. So it is a very convenient way to compare several devices. Moreover, using the common electrical formulation (1), the Q-factor can be computed from measurements and simulations, as well. Then, comparisons between simulations and measurements become possible, which cannot be easily addressed when powers are considered. However, the radiated and dissipated contributions to the overall Q-factor are mixed and cannot be separated when (1) is used. These contributions, as well as the stored energy, are then extracted from EM simulations.

The overall Q-factor of an inductor is commonly defined by the following expression [9]

$$
Q=\frac{2 \omega W_{\text {sto }}}{P_{a c c}}
$$

This definition is consistent with the electrical definition (1). Considering that the accepted power $P_{a c c}$ is resulting from dissipation into materials $\left(P_{\text {diss }}\right)$ and from electromagnetic radiation $\left(P_{\mathrm{rad}}\right)$, as previously stated by $(3)$, the overall $Q$ can be written as follows

$$
Q=\left(\frac{1}{Q_{\text {diss }}}+\frac{1}{Q_{\text {rad }}}\right)^{-1}
$$




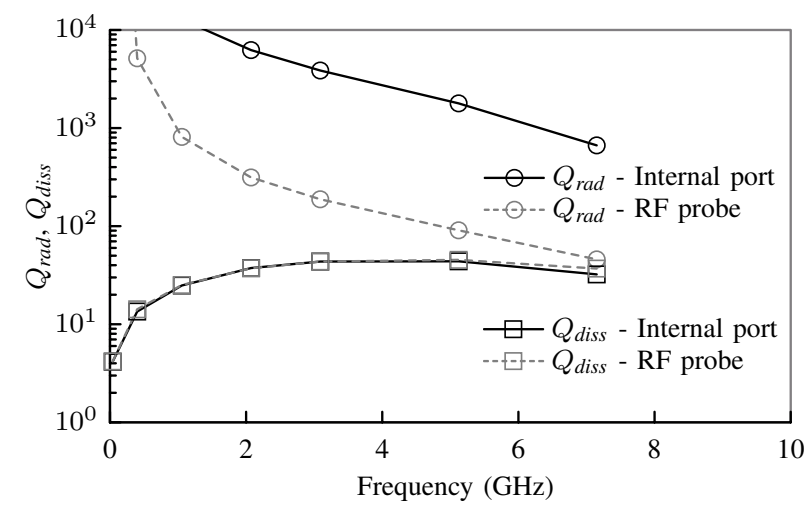

Fig. 10. $Q_{\text {rad }}$ and $Q_{\text {diss }}$ calculated from EM simulations carried out using an internal port (solid lines) and with an RF probe (dashed lines).

where the Q-factors $Q_{\text {diss }}$ and $Q_{\text {rad }}$ are defined relatively to each contribution using (7) and (8), respectively.

$$
\begin{aligned}
Q_{\text {diss }} & =\frac{2 \omega W_{\text {sto }}}{P_{\text {diss }}} \\
Q_{\text {rad }} & =\frac{2 \omega W_{\text {sto }}}{P_{\text {rad }}}
\end{aligned}
$$

It must be noticed that $Q_{r a d}$ value is small when a large radiated power is observed, whereas $Q_{\mathrm{rad}}$ becomes very large when radiations losses cancel out ( $Q$ becomes close to $Q_{\text {diss }}$ ).

\section{$D$. Extraction of $Q_{\mathrm{rad}}$ and $Q_{\mathrm{diss}}$ for the 3-D Solenoid}

Quality factors $Q_{\text {rad }}$ and $Q_{\text {diss }}$, as well as the overall $Q$, are plotted in Fig. 10 and Fig. 11, respectively, for both excitation ways. These graphs show that the overall Q-factor of Fig. 11 results mainly from dissipated losses $\left(Q_{\text {diss }}\right)$ when the inductor is simulated using an internal port: $Q_{\mathrm{rad}}$ remains very high and does not affect the overall quality factor Q. Nevertheless, $Q_{\text {rad }}$ is decreasing largely as the frequency is increasing when the RF probe is taken into consideration. So, from a certain frequency, its influence becomes noticeable on the overall Qfactor, which is well decreased. At $5 \mathrm{GHz}$, this value decreases from 42 to 30 when the RF probe is included, which is consistent with the electrical Q-factor calculated in Fig. 4 and Fig. 7 respectively at the same frequency. In addition, it must be noticed that $Q_{\text {diss }}$ remains unchanged in both cases. Finally, in Fig. 11, the good agreement between calculations from (1) and calculations from (6) confirms that all powers are correctly extracted within simulations.

\section{Accurate On-Wafer Measurements}

The correction of this parasitic probe / DUT coupling effect is not trivial since the radiation intensity is very dependent on the DUT geometry. As a consequence, this effect cannot be simply removed by using any dedicated deembedding structure. The best way is then to avoid these radiations. As pointed out in section III, parasitic radiations result from the difference in potential between the wide metal ring and the external conductor of the feeding coaxial waveguide. The floating metal plate of the probe station chuck acts as a

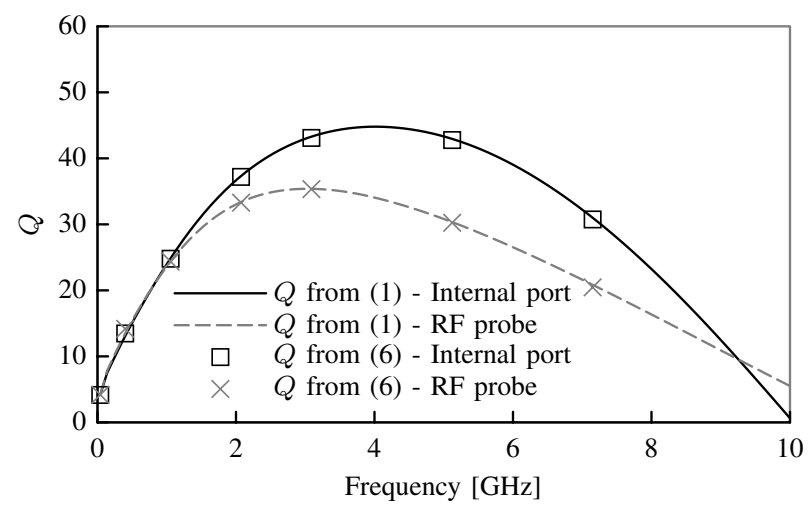

Fig. 11. $Q$ calculated from (1) for both excitation ways (solid and dashed lines) and from (6) (marks).

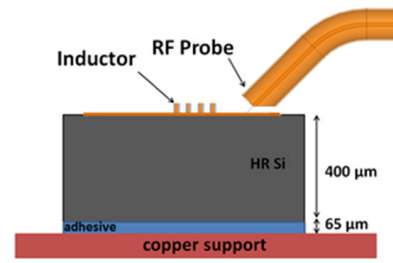

(a)

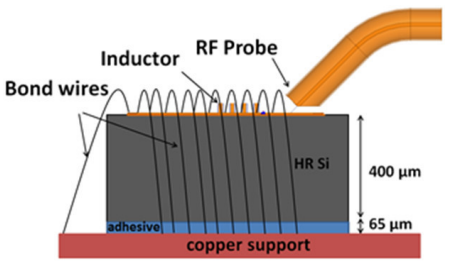

(b)

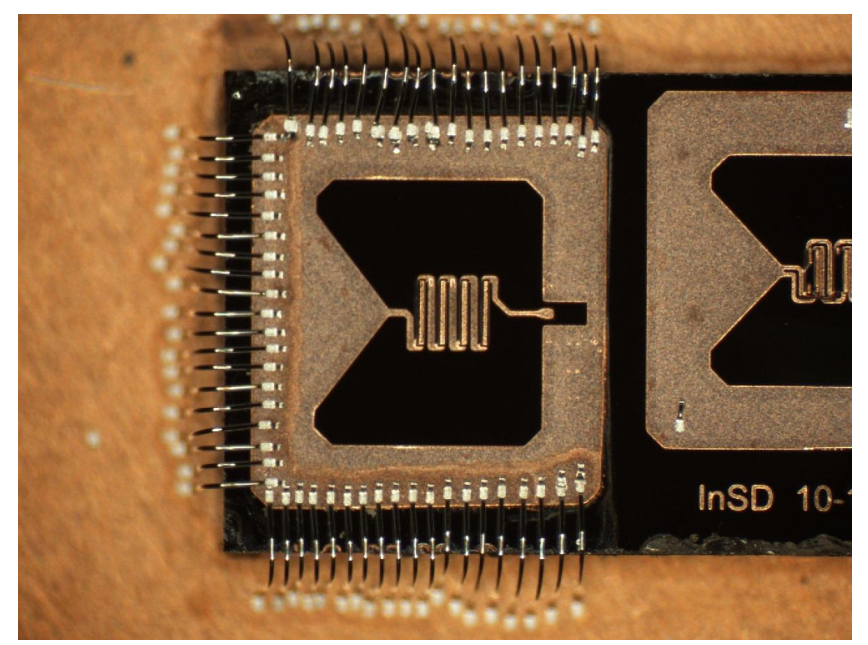

(c)

Fig. 12. (a) Cross-sectional view of the solenoid inductor, (b) wire-bonded to a copper back-plate and (c) the microphotograph of the experiment.

reflector plane for the parasitic dipole antenna. One solution is to short the metal ring of the DUT to the metal plate of the probe station. The experiment is shown in Fig. 12. The DUT is diced and sealed on a large copper plate. The inductor's ground ring is wire-bonded to this metal plate on three of the four sides of the test structure. The DUT placed in this new setup is characterized before and after the wire-bonding.

All measurement results are plotted in Fig. 13. As it can be noticed, the maximum measured Q-factor increases from 35 to 45 and a very good agreement with EM simulations is obtained when wire-bonds connect both metal planes. Moreover, initial EM simulations from Fig. 4, carried out using an internal port, have been added to Fig. 13 and a very good agreement can 

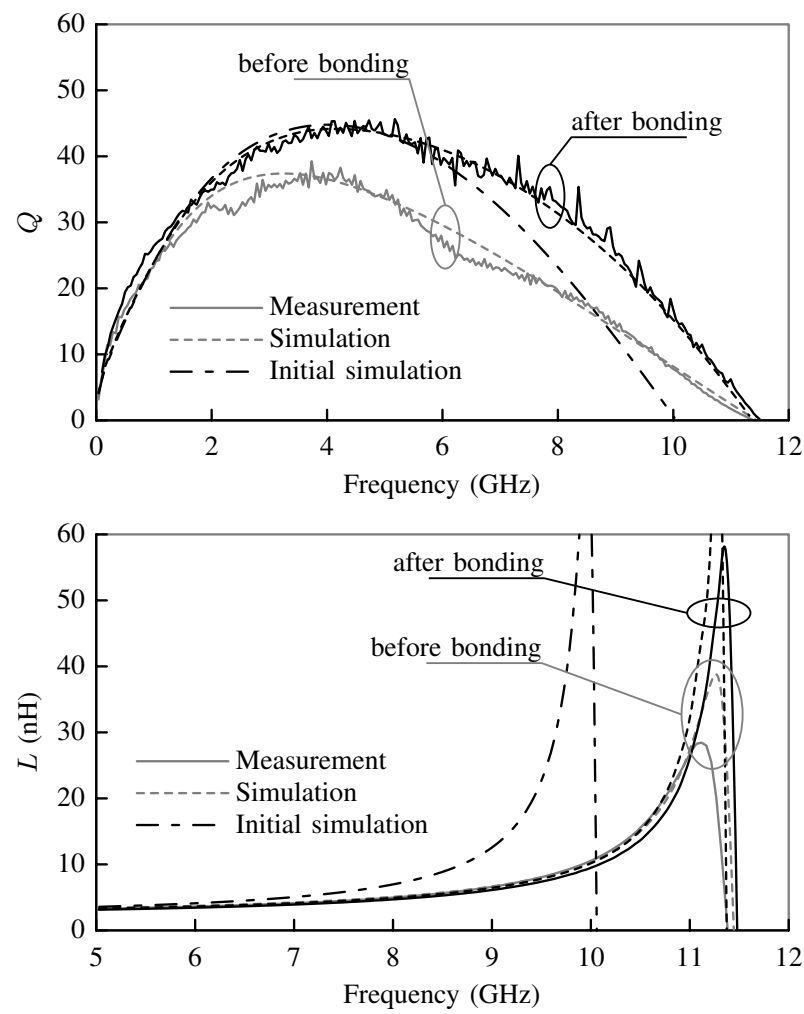

Fig. 13. Measured and simulated Q-factor and inductance value of the solenoid inductor.

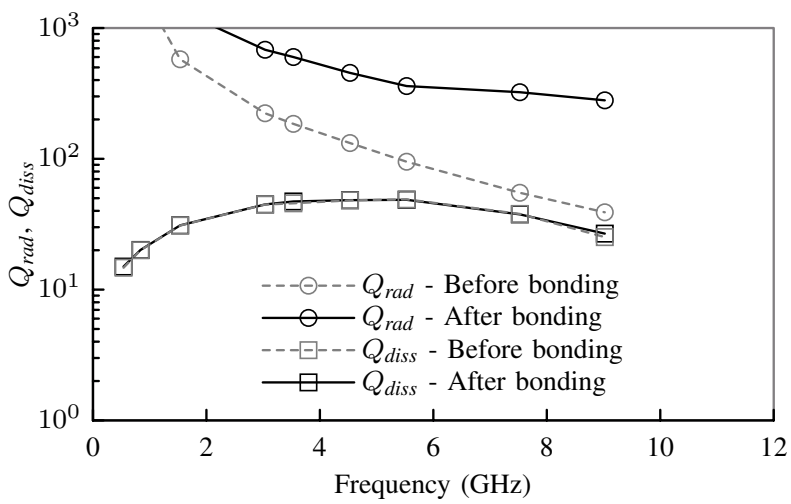

Fig. 14. $Q_{\text {rad }}$ and $Q_{\text {diss }}$ calculated from the new setup before shorting the inductor metal ring (dashed lines) and after (solid lines).

be observed once again, up to $6 \mathrm{GHz}$. Beyond $6 \mathrm{GHz}$, this last agreement becomes worse. The thin layer of adhesive added to stick the inductor on the underlying copper backplate alters the Q-factor and the SRF. This thin dielectric layer increases the distance between the inductor and the backplane and lowers the parasitic capacitor to the ground. Radiation and dissipation Q-factors are extracted from EM simulations before and after bonding (Fig. 14 and Fig. 15). The reduction in radiation losses is confirmed when this new setup is used since $Q_{\text {rad }}$ greatly increases when the metal ring is grounded while $Q_{\text {diss }}$ remains unchanged in both cases.

The above solution may not be suitable for characterizing large series of high-Q inductors. Another way to reduce

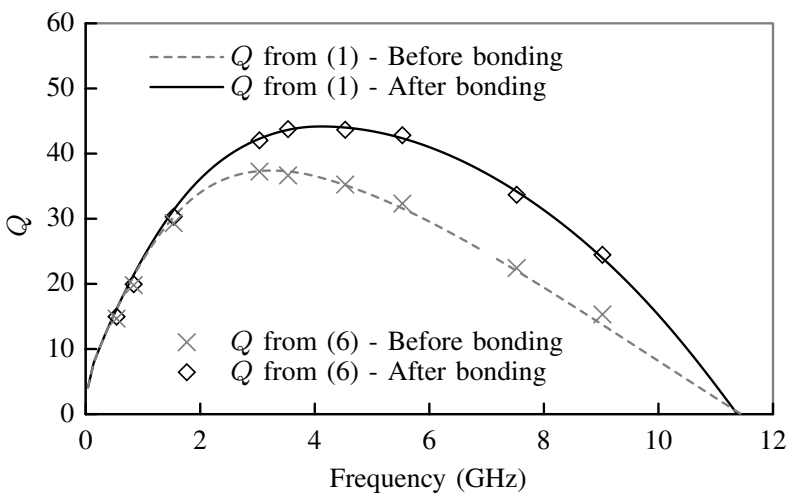

Fig. 15. $Q$ calculated from (1) and (6).

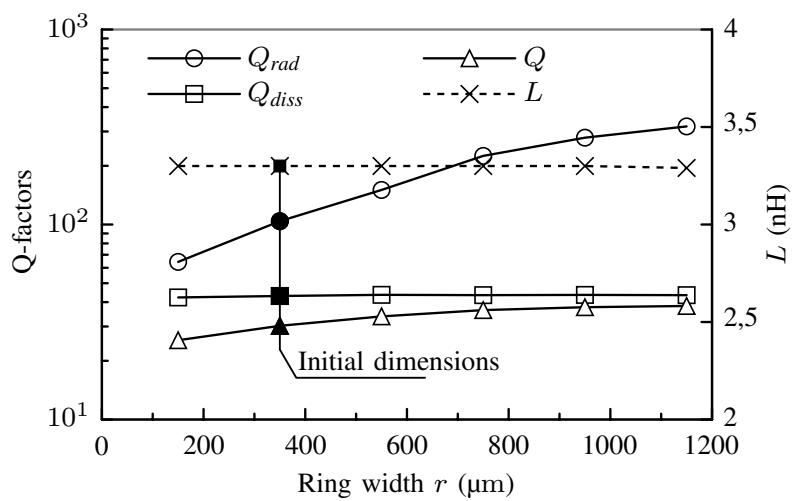

Fig. 16. Effect of the metal ring width $r$ on $Q_{\text {rad }}, Q_{\text {diss }}, Q$ and $L$.

radiation losses must be investigated, which can be directly implemented on wafer. Simulation and calculation techniques introduced in sections III and IV can be used to improve the design of the test structure of the inductor regarding radiation, before fabrication. For this purpose, and as an example, effects on radiation losses of the metal ring dimensions are presented in the case of the 3-D solenoid. The width $r$ of the metal ring and its distance $d$ to the inductor (Fig. 6) have been tuned. Values of $Q_{\text {rad }}, Q_{\text {diss }}, Q$ and $L$ at $5 \mathrm{GHz}$ are plotted versus $r$ and $d$ in Fig. 16 and Fig. 17, respectively. Fig. 16 shows that radiation losses decrease significantly when $r$ is increased since $Q_{\text {rad }}$ widely improves without noticeable change on $Q_{\text {diss }}$ and $L$. From Fig. 17, $Q_{\text {rad }}$ and $Q$ benefit from the reduction of $d$ which improves the confinement of the electromagnetic field into the device. However, such containment now slightly reduces the inductor value $L$. As a conclusion, the designer can set $d$ and $r$ that reach the best trade-off.

\section{CONCLUSION}

A parasitic radiative interaction is identified between the RF probe and the on-wafer measured structure. It is observed on planar and 3-D on-chip inductors fabricated on high-resistivity or insulating substrates and when $Q$ is exceeding a value of 25. Radiation losses occurring on these devices are leading to experimental Q-factors well under simulated values. However, this effect is reproduced and quite accurately quantified using EM simulations when an RF probe model is included for 


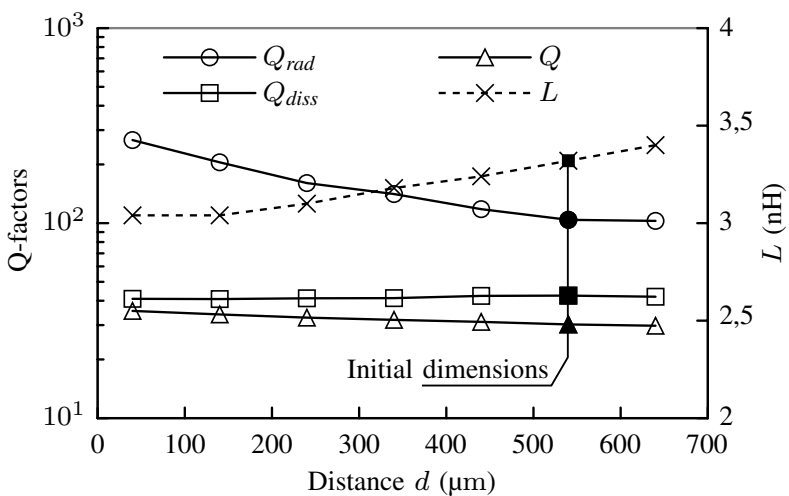

Fig. 17. Effect of the distance $d$ between the metal ring and the inductor on $Q_{\text {rad }}, Q_{\text {diss }}, Q$ and $L$.

the test signal feeding of the high-Q structure. To cancel these radiations, a new experiment is designed by shorting the metal ring of the DUT to the underneath metal plate. The measurements performed on this new test structure confirm the large reduction in radiation losses and the measured Q-factor now agrees very well with the performance initially predicted by EM simulations. We also demonstrate that most of these radiations may be avoided by properly setting dimensions of the metal ring surrounding the inductor.

\section{ACKNOWLEDGMENT}

The authors would like to thank Pr. Damienne Bajon, from the Institut supérieur de l'aéronautique et de l'espace (ISAE), Toulouse, for fruitful discussions and Alexandre Rumeau, from the Laboratory of Analysis and Architecture of Systems (LAAS), Toulouse, for technical support in RF measurements.

\section{REFERENCES}

[1] W. Kuhn and A. Boutz, "Measuring and Reporting High Quality Factors of Inductors Using Vector Network Analyzers," IEEE Trans. Microw. Theory Techn., vol. 58, no. 4, pp. 1046-1055, Apr. 2010.

[2] J. C. Rautio and R. Groves, "A potentially significant on-wafer highfrequency measurement calibration error," IEEE Microwave Magazine, vol. 6, no. 4, pp. 94-100, Apr. 2005.

[3] A. M. E. Safwat, "Study of microstrip mode in RF on-wafer probes," Microwave and Optical Technology Letters, vol. 45, no. 4, pp. 324-328, Apr. 2005.

[4] B. Rejaei, A. Akhnoukh, M. Spirito, and L. Hayden, "Effect of a Local Ground and Probe Radiation on the Microwave Characterization of Integrated Inductors," IEEE Trans. Microw. Theory Techn., vol. 55, no. 10 , pp. 2240-2247, Oct. 2007.

[5] C. Andrei, D. Gloria, F. Danneville, P. Scheer, and G. Dambrine, "Coupling on-wafer measurement errors and their impact on calibration and deembedding up to $110 \mathrm{GHz}$ for CMOS millimeter wave characterizations," in IEEE International Conference on Microelectronic Test Structures, ICMTS '07, 2007, pp. 253-256.

[6] K. Mohammadpour-Aghdam, S. Brebels, A. Enayati, R. Faraji-Dana, G. A. E. Vandenbosch, and W. DeRaedt, "RF probe influence study in millimeter-wave antenna pattern measurements," Int. J. RF and Microwave Comp. Aid. Eng., vol. 21, no. 4, pp. 413-420, Jul. 2011.

[7] A. Ghannam, D. Bourrier, L. Ourak, C. Viallon, and T. Parra, "3D Multilayer Copper Interconnects for High-Performance Monolithic Devices and Passives," IEEE Trans. Compon., Packag., Manuf. Technol., vol. 3, no. 6, pp. 935-942, Jun. 2013.

[8] D. M. Pozar, Microwave Engineering, 4th ed. New York: John Wiley \& Sons, 2011.

[9] K. O, "Estimation methods for quality factors of inductors fabricated in silicon integrated circuit process technologies," IEEE J. Solid-State Circuits, vol. 33, no. 8, pp. 1249-1252, Aug. 1998.

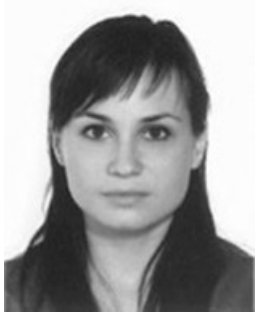

Olga Bushueva received the M.S. degree in telecommunication engineering from the Moscow Technical University of Communications and Informatics, Moscow, Russia, in 2007, and the M.S. degree in microwave devices and modeling from the University of Brest (UBO), Brest, France, in 2012.

She is currently working toward the $\mathrm{PhD}$ degree at LAAS-CNRS and University of Toulouse III, Toulouse, France. Her current research interests include process development and optimization for the integration of passive devices. She designed high$\mathrm{Q}$ power inductors/transformers and developed an above-IC process for their integration.

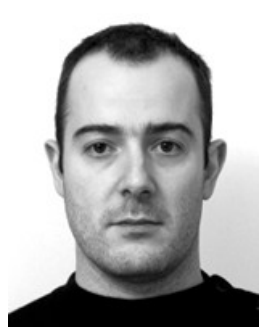

Christophe Viallon (M'14) received the M.S. and $\mathrm{Ph} . \mathrm{D}$. degrees in electrical engineering from University of Toulouse III, Toulouse, France, in 1999 and 2003, respectively.

In 2005 , he has held a post-doctoral position to study nonlinear noise inside cellular power amplifiers with the Radio Product Division at Freescale Semiconductors, Toulouse, France. He is now an associate Professor at the University of Toulouse III since 2005 and is conducting researches with the Laboratory of Analysis and Architecture of Systems, French National Research Agency. His technical interests and expertise are in the field of passive devices design, modeling and high-frequency characterization as well as analog and RF IC design in bipolar and CMOS technologies up to millimeter-wave frequencies. He is also involved in the development of a low-cost above-IC fabrication process for innovative inductive components and interconnections.

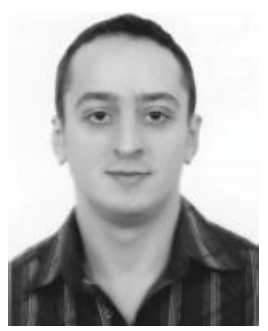

Ayad Ghannam received $\mathrm{Ph} . \mathrm{D}$ degree in $\mathrm{Mi}$ crowave, Electromagnetism and Optoelectronic from University of Toulouse III, France, in 2010 .

During Ph.D, he worked as a research engineer at Freescale Semiconductors, France branch, and LAAS-CNRS where he designed high-Q power inductors and developed an Above-IC process for their integration. After graduation, he continued working at LAAS-CNRS as a research engineer where he developed an innovative 3-D interconnection technology. He founded 3DiS Technologies in 2014, a spinoff that offers innovating packaging and system integration solutions based on a novel 3-D RDL technology. His current research interests include high$\mathrm{Q}$ power inductors / transformers, filters / duplexers, baluns, micro-antennas, 3-D interconnection, 3-D system integration and 3-D wafer-level packaging.

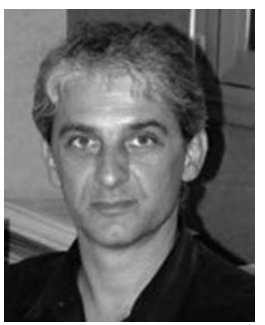

Thierry Parra was born in August 1964. He received the Ph.D. degree in electrical engineering from University of Toulouse III, Toulouse, France, in 1991 .

He joined the Laboratory of Analysis and Architecture of Systems, French National Research Agency in 1991, and since 2002, he has been a Professor of electrical engineering. His current research interests include microwave component and circuit integration from RF up to the millimeter wave frequency range, optimization and design of microwave integrated circuit (using either GaAs PHEMT technologies or SiGe BiCMOS or CMOS technologies), as well as technological developments for innovative inductive components and interconnections. 